Vol. 5, No. 1, 2014

\title{
Determination of the Concentration and Isotope Ratio of Uranium in Soil and Water by Thermal Ionization Mass Spectrometry
}

\author{
Jong-Ho Park*, Sujin Park ${ }^{\dagger}$, and Kyuseok Song \\ Nuclear Chemistry Research Division, Korea Atomic Energy Research Institute, Daejeon 305-353, Korea
}

Received December 5, 2013; Accepted December 10, 2013

First published on the web March 30, 2014; DOI: 10.5478/MSL.2014.5.1.12

\begin{abstract}
Thermal ionization mass spectrometry (TIMS) was used to determine the concentration and isotope ratio of uranium contained in samples of soil and groundwater collected from Korea. Quantification of uranium in ground water samples was performed by isotope dilution mass spectrometry. A series of chemical treatment processes, including chemical separation using extraction chromatography, was applied to the soil samples to extract the uranium. No treatments other than filtration were applied to the groundwater samples. Isotopic analyses by TIMS showed that the isotope ratios of uranium in both the soil and water samples were indistinguishable from those of naturally abundant uranium. The concentration of uranium in the groundwater samples was within the U.S. acceptable standards for drinking water. These results demonstrate the utility of TIMS for monitoring uranium in environmental samples with high analytical reliability.
\end{abstract}

Key words: Uranium, TIMS, Isotopic Analysis, Environmental Monitoring

\section{Introduction}

As a primordially occurring element, uranium exists in the environment at trace levels. For example, the normal concentration of uranium in soil ranges from $300 \mu \mathrm{g} / \mathrm{kg}$ to $11.7 \mathrm{mg} / \mathrm{kg}$. ${ }^{1}$ The natural uranium concentration in water is less than that of soil due to the low solubility of uranium compounds. The U.S. EPA (United States Environment Protection Agency) standard for uranium in drinking water is $30 \mu \mathrm{g} / \mathrm{L}{ }^{2,3}$ Concentrations of uranium in typical Korean drinking water are an order of magnitude lower. ${ }^{4}$ Since the environmental uranium content is of public interest and is a primary indicator of the safety of nuclear power plants, it is important to reliably monitor uranium concentrations and isotope ratios in environmental samples.

Monitoring uranium content and isotope ratios in environmental samples is typically performed using alpha spectrometry and inductively coupled plasma atomic

\section{Open Access}

*Reprint requests to Jong-Ho Park

E-mail: jongho@kaeri.re.kr

${ }^{\dagger}$ Current Address: CKD Research Institute, Yong-In 446-916, Korea

All MS Letters content is Open Access, meaning it is accessible online to everyone, without fee and authors' permission. All MS Letters content is published and distributed under the terms of the Creative Commons Attribution License (http://creativecommons.org /licenses/by/3.0/). Under this license, authors reserve the copyright for their content; however, they permit anyone to unrestrictedly use, distribute, and reproduce the content in any medium as far as the original authors and source are cited. For any reuse, redistribution, or reproduction of a work, users must clarify the license terms under which the work was produced. emission spectrometry (ICP-AES). However, due to the relatively low sensitivity of these techniques for uranium, a large number of samples and long measurement times are generally required to obtain reliable results. Moreover, the accuracy of alpha spectrometry allows only a rough estimation of uranium levels. Mass spectrometry, which boasts both high sensitivity and accuracy, is considered one of the best alternatives to the aforementioned methods. One drawback of mass spectrometry, however, is interference from other elements in a sample or solution matrix, which can distort or skew the actual concentrations and/or isotope ratios. ${ }^{5,6}$ Therefore, complicated chemical treatments or separation processes are generally required to remove interfering elements prior to mass spectrometric measurements.

Thermal ionization mass spectrometry (TIMS) is a powerful technique that can be used to determine the isotopic ratios of trace elements, including uranium, contained in the environment with high sensitivity, accuracy, and precision. ${ }^{7-10}$ The high analytical reliability of TIMS has led to its widespread use in geology, forensic science, and nuclear safeguards. ${ }^{9,11-13}$ The total amount of a given element, as opposed to just the isotopic ratio,can be measured by combining TIMS with isotope dilution mass spectrometry (IDMS). This combined system has become known as ID-TIMS. ${ }^{14}$

Elemental interference in TIMS measurements is relatively low because no additional gas is needed, as in inductively coupled plasma mass spectrometry (ICP-MS), and because of the complete dissociation of elements in the loaded sample. Other matrix effects and mass bias are also 
low in TIMS measurements. ${ }^{7,8,10}$ Moreover, differences in the evaporation temperatures of the elements allows them to be separated as a function of time. ${ }^{15}$ For example, plutonium oxide $\left(\mathrm{PuO}_{2}\right)$ melts and evaporates at a lower temperature than uranium dioxide $\left(\mathrm{UO}_{2}\right)$. Therefore, in a TIMS experiment with a sample containing these two compounds, the plutonium signal will appear before that of uranium.

This report details the determination of uranium isotope ratios in soil and groundwater samples collected from Korea using TIMS. Total uranium concentrationswere also determined for the groundwater samples using ID-TIMS. TIMS isotopic measurements in soil samples were preceded by chemical treatment and separation. No treatments other than filtration were applied to the groundwater samples. This study demonstrates the utility of TIMS for the determination of uranium concentration and isotope ratios in environmental samples.

\section{Experimental}

Soil samples were collected from two sites in Daejeon, Korea. Samples were acquired from $\sim 30 \mathrm{~cm}$ below ground to avoid interference from recent contamination. The two sites were $\sim 3 \mathrm{~km}$ from each other and at least $2 \mathrm{~km}$ from the Hanaro research reactor of the Korea Atomic Energy Research Institute (KAERI).

Organic materials in the samples were decomposed by baking at over $510^{\circ} \mathrm{C}$ for 10 hours in a muffle furnace (DF3.5, Daeheung Science). Acid digestion of inorganic materials in the decomposed samples was performed with concentrated nitric acid $(8 \mathrm{M})$ and hydrofluoric acid at $\sim 80^{\circ} \mathrm{C}$ for $3 \mathrm{~h}$. The samples were then filtered to remove residue and dried. After drying, $10 \mathrm{~mL}$ of $3 \mathrm{M}$ nitric acid were added to each sample.

The samples were loaded onto ion exchange columns filled with UTEVA resin (Eichrom). Sequential washes with $8 \mathrm{M}$ nitric acid, $9 \mathrm{M}$ hydrochloric acid, and $5 \mathrm{M}$ hydrochloric acid were performed to remove americium, plutonium, and neptunium, respectively. An elution with weak hydrochloric acid $(0.01 \mathrm{M})$ was used to collect pure uranium. The sample matrix was then changed to a nitric acid solution by allowing the hydrochloric acid to evaporate and adding $1 \mathrm{M}$ nitric acid. The chemical treatments of the soil samples are summarized in Figure 1. No further treatment to determine the amounts of uranium in the soil samples was applied in this study since considerable degree of sediment was observed, which would distort content information unless complete physical removal of the sediment was performed prior to sample weighing.

Water samples were collected from a hot spring in Daejeon and from an agricultural field in Sang-Ju, Korea. Since the expected mass spectrometric interference in the water samples was low, no chemical treatments or separations

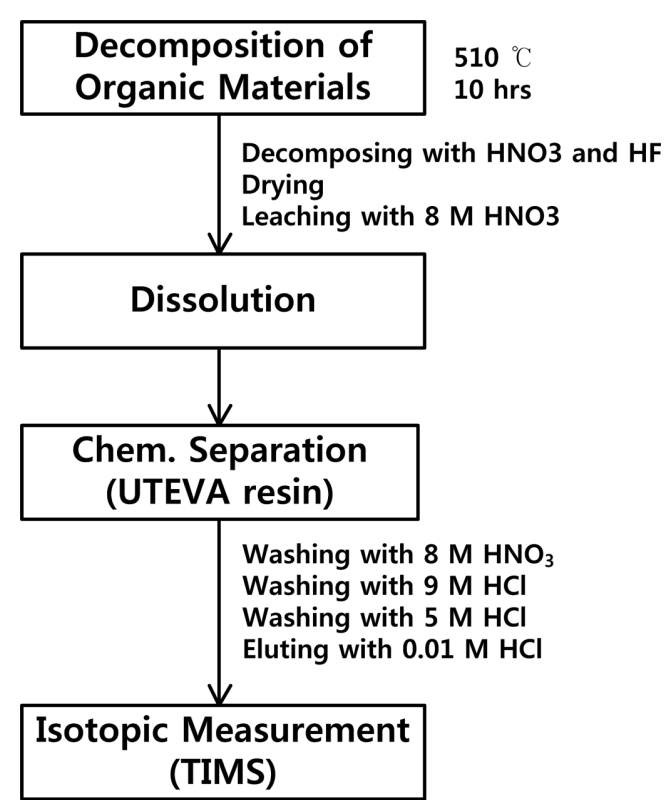

Figure 1. Chemical treatment and processing of soil samples.

other than filtration were performed prior to the TIMS measurements. The samples were divided into two portions. To quantitate the uranium content of the samples, appropriate amounts of IRMM040a (a reference material) were spiked into an aliquot of each water sample. The remainder of each sample was used to determine the isotopic ratios of uranium. The volume of the groundwater samples was reduced by a factor of ten to increase the uranium concentration to a more reliably measured level. No such enrichment was performed for the hot spring water sample.

One microliter of each sample was loaded onto individual rhenium filaments (zone-refined, Thermo Scientific) and fixed at $1.8 \mathrm{~A}$ for $30 \mathrm{~s} .^{16,17}$ The uranium background of each filament had been minimized prior to use. The system was pumped for at least $5 \mathrm{~h}$ after loading the filaments and prior to acquiring TIMS data.

A thermal ionization mass spectrometer (TRITON, Thermo Scientific) was used to determine the uranium isotopic ratios of the samples. The experimental setup has been described in detail elsewhere. ${ }^{16,17}$ Multi-dynamic measurements utilizing a secondary electron multiplier (SEM) and Faraday cups were used with the soil samples and the hot spring water sample. Dynamic measurements, in which the magnetic field was regulated using an SEM to receive ion signals from each individual isotope, were used with the ground water sample. The continuous heating method, which is a modified total evaporation method, was adopted to evaporate the samples. ${ }^{18}$ Additional isotopic measurements of a reference material (NIST U010) were performed to correct for mass bias as described in detail in a previous study. Isotopic ratios are expressed with uncertainties as estimated based on GUM (Guide to the expression of Uncertainty in Measurements) ${ }^{19}$. 
Table 1. The isotopic ratios of uranium in soil samples.

\begin{tabular}{ccc}
\hline \hline & $\begin{array}{c}\mathrm{n}\left({ }^{234} \mathrm{U}\right) / \mathrm{n}\left({ }^{238} \mathrm{U}\right) \\
\left(\times 10^{-5}\right)\end{array}$ & $\begin{array}{c}\mathrm{n}\left({ }^{235} \mathrm{U}\right) / \mathrm{n}\left({ }^{238} \mathrm{U}\right) \\
\left(\times 10^{-3}\right)\end{array}$ \\
\hline Natural Abundance & $5-6$ & $7.198-7.202$ \\
Soil \#1 & $5.75[ \pm 0.11]$ & $7.281[ \pm 0.066]$ \\
Soil \#2 & $6.01[ \pm 0.10]$ & $7.260[ \pm 0.059]$ \\
\hline
\end{tabular}

Numbers in brackets indicate expanded uncertainties $U=k u_{c}$

\section{Results and discussion}

The isotopic ratios of uranium contained in the soil samples are summarized in Table 1. Typical isotopic ratios of naturally occurring uranium are also shown for comparison. The isotopic ratios of $n\left({ }^{235} \mathrm{U}\right) / \mathrm{n}\left({ }^{238} \mathrm{U}\right)$ in the two soil samples were $7.281[ \pm 0.066] \times 10^{-3}$ and $7.260[ \pm 0.059] \times 10^{-3}$, which are indistinguishable from the isotopic ratio of naturally occurring uranium. The isotopic ratios of ${ }^{234} U\left(\right.$ as $\left.n\left({ }^{234} U\right) / n\left({ }^{238} U\right)\right)$ in the two samples were $5.75[ \pm 0.11] \times 10^{-5}$ and $6.01[ \pm 0.10] \times 10^{-5}$, which fall within the range of natural abundance ratios $\left(5 \times 10^{-5}\right.$ to $6 \times 10^{-5}$ ).

Although not listed in Table $1, n\left({ }^{236} U\right) / n\left({ }^{238} U\right)$ ratios in the soil samples were on the order of $10^{-7}$. The natural abundance ratio of ${ }^{236} \mathrm{U}$ is negligible or on the order of $10^{-12}$. The small signal intensity at $236 \mathrm{amu}$ in the current study was due to peak tailing effects by the major isotopes $\left({ }^{235} \mathrm{U}\right.$ and ${ }^{238} \mathrm{U}$ ) in the mass spectra. In addition, isobaric interference from lead oxides such as ${ }^{204} \mathrm{PbO}_{2}$ can contribute to the signal intensity at 236 amu. Preliminary isotopic ratio measurements on a certified reference material with a natural abundance (CRM-112A from the New Brunswick Laboratory) showed that the limit of detection of $n\left({ }^{236} \mathrm{U}\right) / \mathrm{n}\left({ }^{238} \mathrm{U}\right)$ using the technique described herein was $6 \times 10^{-7}$. Therefore, the observed $n\left({ }^{236} \mathrm{U}\right) / \mathrm{n}\left({ }^{238} \mathrm{U}\right)$ ratio in the soil samples was deemed indistinguishable from that of naturally occurring uranium.

While the amount of uranium in the soil samples was not quantitated in the current study, isotope dilution mass spectrometry (IDMS) was used to determine the uranium concentration in the water samples. The samples were spiked with an internal standard of ${ }^{233} \mathrm{U}$ from reference material IRMM-040a. Concentration was calculated as

$$
c\left({ }^{238} U, x\right)=\frac{R_{y}-R_{b}}{R_{b}-R_{x}} \cdot \frac{1}{R_{y}} \cdot \frac{m_{y}}{m_{x}} \cdot c\left({ }^{233} U, y\right)
$$

where,

$$
R_{b} \text { : amount ratio } \mathrm{n}\left({ }^{233} \mathrm{U}\right) / \mathrm{n}\left({ }^{238} \mathrm{U}\right) \text { in the blend }
$$

$R_{x}$ : amount ratio $\mathrm{n}\left({ }^{233} \mathrm{U}\right) / \mathrm{n}\left({ }^{238} \mathrm{U}\right)$ in the sample
$R_{y}$ : amount ratio $\mathrm{n}\left({ }^{233} \mathrm{U}\right) / \mathrm{n}\left({ }^{238} \mathrm{U}\right)$ in the spike

$m_{x}$ : mass of the sample

$m_{y}:$ mass of the spike

$c\left({ }^{238} U, x\right)$ : amount of ${ }^{238} \mathrm{U} \mathrm{kg}^{-1}$ in the sample

$c\left({ }^{233} U, y\right)$ : amount of ${ }^{233} \mathrm{U} \mathrm{kg}^{-1}$ in the spike

The uranium concentration in the hot spring water was $120.05[ \pm 0.70] \mu \mathrm{g} / \mathrm{L}$, which agrees with the concentrations measured using a kinetic phosphorescence analyzer (KPA). This concentration is higher than the reported value for normal underground water in the Daejeon area, which is $\sim 80 \mu \mathrm{g} / \mathrm{L} .{ }^{4}$ However, the uranium concentration in hot spring water is generally higher than in normal underground water. $^{20}$ Therefore, the isotopic ratios of uranium were in agreement with those of naturally occurring uranium.

The groundwater samples contained $0.1487[ \pm 0.0012] \mu \mathrm{g} /$ $\mathrm{L}$ of uranium, which is below the U.S. standard for drinking water $(30 \mu \mathrm{g} / \mathrm{L}){ }^{2,3}$ The measured ratio of $\mathrm{n}\left({ }^{234} \mathrm{U}\right) / \mathrm{n}\left({ }^{238} \mathrm{U}\right)$ was slightly higher than that of naturally occurring uranium, although this discrepancy is likely an artifact of the low intensity of the ${ }^{234} \mathrm{U}$ signal in the mass spectrum. The abundance of ${ }^{235} \mathrm{U}$ coincided with that of naturally occurring uranium. Negligible signal intensities of ${ }^{236} \mathrm{U}$ were observed in both of the water samples. The concentrations and isotope ratios of uranium contained in the groundwater samples are summarized in Table 2.

\section{Summary}

TIMS was used in isotopic analyses of soil and water samples collected from Korea. Soil samples were chemically digested and subject to extraction chromatography using UTEVA resin to separate the uranium from the other elemental constituents. Since elemental interference in TIMS measurements on the water samples was expected to be negligible, no chemical treatments were performed on the groundwater samples prior to analysis. The isotopic ratios of uranium in the samples agreed with those of naturally occurring uranium. The uranium concentration of the ground water samples was below the U.S. EPA standard for drinking water. The results given herein demonstrate the utility of TIMS for monitoring uranium in environmental samples with high analytical reliability.

\section{Acknowledgements}

The authors acknowledge the financial support for this study from the Nuclear Safety and Security Commission of the Korean government.

Table 2. The concentration and isotopic ratios of uranium in groundwater samples.

\begin{tabular}{cccc}
\hline \hline & Concentration $(\mu \mathrm{g} / \mathrm{L})$ & $\mathrm{n}\left({ }^{234} \mathrm{U}\right) / \mathrm{n}\left({ }^{238} \mathrm{U}\right)\left(\times 10^{-5}\right)$ & $\mathrm{n}\left({ }^{235} \mathrm{U}\right) / \mathrm{n}\left({ }^{238} \mathrm{U}\right)\left(\times 10^{-3}\right)$ \\
\hline Hot Spring Water & $120.05[ \pm 0.70]$ & $5.94[ \pm 0.15]$ & $7.231[ \pm 0.037]$ \\
Ground water & $0.1487[ \pm 0.0012]$ & $8.4[ \pm 1.3]$ & $7.30[ \pm 0.10]$ \\
\hline
\end{tabular}

Numbers in brackets indicate expanded uncertainties $U=k u_{c}$ 


\section{References}

1. United Nations, United Nations Scientific Committee on the Effect of Atomic Radiation; New York, 1993.

2. U.S. EPA (United States Geological Survey), Integrated risk information system (IRIS) on uranium, soluble salt, Cincinnati, 1993.

3. USGS (United States Geological Survey), Occurrence of selected radionuclides in ground water used for drinking water in the United States, 2001

4. Kim, B.; Cho, G.; Kim. S. The Annual Report of Busan Metropolitan city Institute of Health \& Environment, 2012, 22, 91.

5. Schoenberg, R.; von Blanckenburg, F. Int. J. Mass Spectrom. 2005, 242, 257.

6. Chan, G. C.-Y.; Hieftje, G. M. Spectrochim. Acta Part B 2006, 61, 642 .

7. Cohen, A. S.; Belshaw, N. S.; O'Nions, R. K. Int. J. Mass Spectrom. Ion Process 1992, 116, 71.

8. Heumann, K. G.; Eisenhut, S.; Gallus, S.; Hebeda, E. H.; Nusko, R.; Vengosh, A.; Walczyk, T. Analyst 1995, 120, 1291.

9. Rubin, K. H. Chemical Geology 2001, 175, 723.

10. Richter, S.; Goldberg, S. A. Int. J. Mass Spectrom. 2003,
$229,181$.

11. Donohue, D. L. J. Alloy Compd. 1998, 271-273, 11.

12. Stetzer, O.; Betti, M.; van Geel, J.; Erdmann, N.; Kratz, J.; Schenkel, R.; Trautmann, N. Nuclear Inst. and Methods in Physics Research A 2004, 525, 582.

13. Kraiem, M.; Richter, S.; Kuhn, H.; Stefaniak, E. A.; Kerckhove, G.; Truyens, J.; Aregbe, Y. Anal. Chem. 2011, 83, 3011.

14. Aggarwal, S. K.; Chourasiya, G.; Duggal, R. K.; Raoi, R.; Jain, H. C. Int. J. Mass Spectrom. Ion Process 1986, 69, 137.

15. Lee, C.; Suzuki, D.; Saito-Kokubu, Y.; Esaka, F.; Margara, M.; Kimura, T. Int. J. Mass Spectrom. 2012, 314, 57.

16. Park, J.; Choi, I.; Park, S.; Lee, M.; Song, K. Bull. Korean Chem. Soc. 2011, 32, 4327.

17. Park, J.; Choi, I.; Song, K. Mass Spectrom. Lett. 1, 1, 17.

18. Suzuki, D.; Kokubu, Y. S.; Sakurai, S.; Lee, C. G.; Magara, M.; Iguchi, K.; Kimura, T., Int. J. Mass. Spectrom. 2010, 294, 23.

19. Bürger, S.; Essex, R. M.; Mathew, K. J.; Richter, S.; Thomas, R. B. Int. J. Mass Spectrom. 2010, 294, 65.

20. Lee, M. H.; Choi, G. S.; Cho, Y. H.; Lee, C. W.; Shin, H. S. J. Environ. Radioact. 2001, 57, 105. 